\title{
Robust force and torque measurements by integrated sensors in hollow shafts
}

\author{
Martin Krech ${ }^{1}$, Peter Groche ${ }^{1}$ \\ 1 Institute for Production Engineering and Forming Machines, Otto-Berndt-Straße 2, 64287 Darmstadt, \\ krech@ptu.tu-darmstadt.de
}

\begin{abstract}
:
This contribution presents a manufacturing method for producing hollow shafts with integrated sensors. An incremental forming process is used to join a deformation sensing element in a tubular part during forming. A mechanical pre-tension is created to ensure a high linearity. The sensor signals are utilized during manufacturing to monitor and control the processes. Control and monitoring strategies are derived in order to achieve a constant quality of the sensory structures. In order to design the joining process a numerical model is used. The resulting pre-tension force could be predicted numerically with a deviation of $30 \%$ for aluminum and $6 \%$ for the steel tubes. A $32 \times 4 \mathrm{~mm}$ hollow shaft is produced and characterized with $\pm 1.2 \mathrm{kNm}$ on a torque testing machine. A demonstrator with an integrated miniature measurement amplifier illustrates the high potential of measuring at the inside of rotating or moving parts to exploit inaccessible measurement regions.
\end{abstract}

Key words: sensor integration, FEM, process control, calibration, torque measurement

\section{Introduction}

Typical mechanical sensors consist of a deformation element and superficially attached strain gauges. While high-precision sensors are especially designed regarding their materials and geometry, also standard components can be upgraded to sensors [1]. However, in case of rotating and moving objects, it is necessary to attach an electronic unit to the part for amplification and transmission of the sensor signals. This requires additional space at the outer surface of the part. This fact often causes difficulties and restrictions in the implementations. For instance, if the regions are exposed to severe environmental conditions or come in contact with other objects and humans. It is therefore desirable to place sensors at the inside of mechanical parts. However, the limited accessibility generally hinders a bonding at the inside and a subsequent connection to the electronic unit is often impossible.

Thus, a process is presented which aims to integrate deformation sensing elements into hollow tubular parts [2]. Consequently, the deformation element is protected from environmental influences and can be optimized regarding its sensitivity, linearity and robustness against parasitic loads. Additionally, a higher degree of freedom to design and integrate transducers in machines, plants and everyday objects arises. Beside the advantages of the higher integration level also the manufacturing process can benefit from an early fusion of the mechanical and electronic parts.

Conventional manufacturing processes of monolithic sensors require the mechanical structure to be in its final form, before merging with the sensor and electronic components, Fig 1 (top). Additional forming and machining operations are usually impeded by the exposed sensor elements at the outer surface. Therefore, all necessary process monitoring and control in the manufacturing process of the mechanical parts has to be realized using external sensors. After the application a coating or casing is necessary to protect the sensitive sensor and electronic elements. The final step of the procedure is to calibrate the sensory structure. For this, it has to be clamped on another machine which is an additional time investment.

A novel approach aims for a higher integration level and an earlier fusion of the sensory and electronic components, Fig 1 (bottom). The deformation sensing elements are protected at the inside of the tubular part ab initio. Thus, several process steps of coating and protection can be simplified or left out. Additionally, the possibility to utilize the sensory functionality throughout the process emerges. Instead of using external sensors for monitoring and controlling the processes, the integrated sensing elements are utilized for these tasks. 
The deformation sensing element is located very close to the interaction zone of the process and is therefore predestinated to gain direct information out of each process step.

Another promising approach is the determination of the sensitivity of low-accuracy sensors directly during the manufacturing process. The tool strokes, which form the part in its final form, are utilized to establish the link between the applied loads and the sensitivity of the sensor. For this, additional reference sensors are equipped to the forming machine in order to calibrate the sensory structure efficiently without additional process steps and handling.
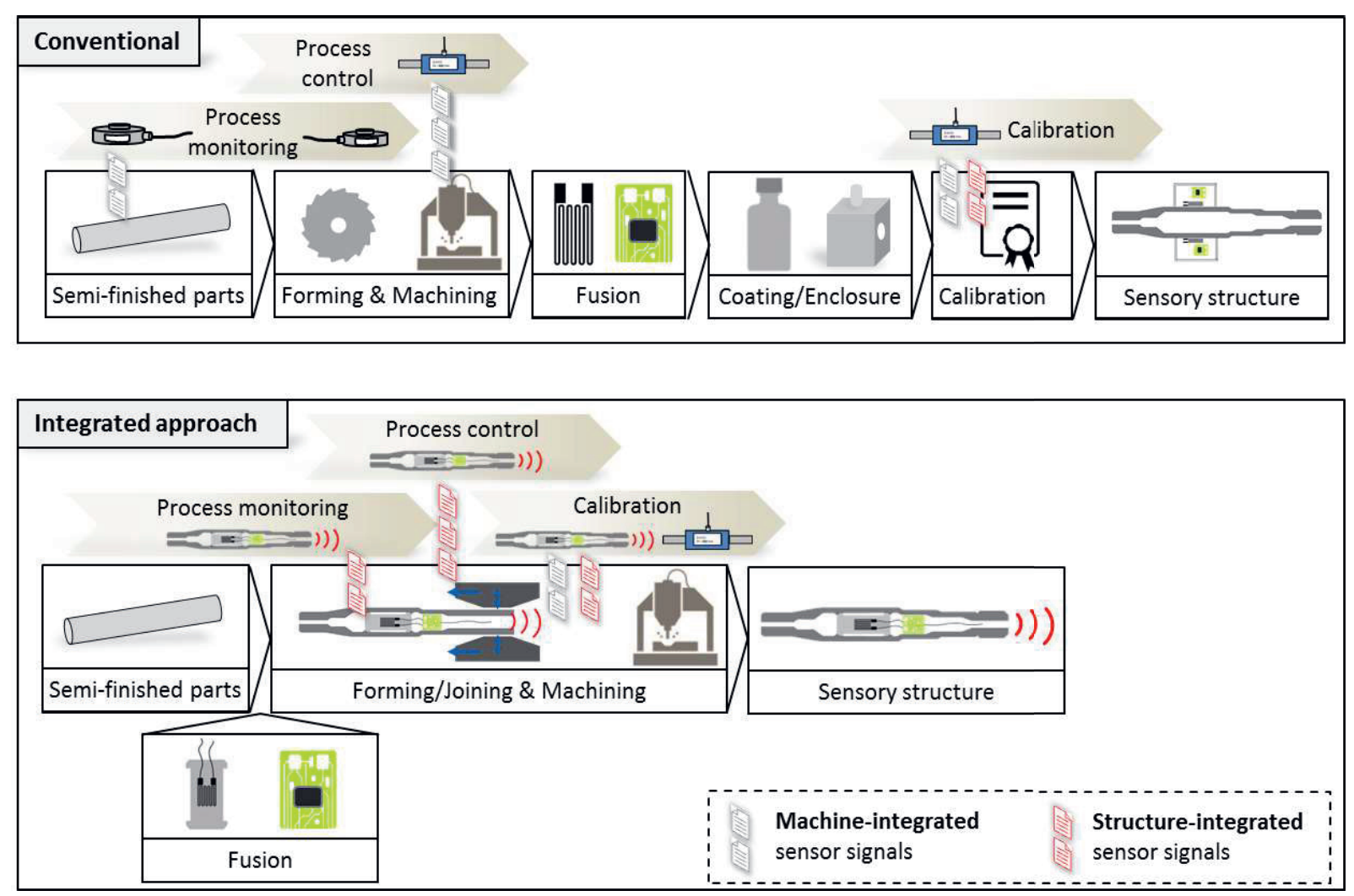

Fig.1. Comparison of a conventional and the integrated manufacturing sequence of mechanical sensors.

The main challenge of integrating sensor elements during a forming process is to avoid damaging of the sensitive elements. Furthermore, a mechanical pre-tension has to be generated to ensure the desired properties of the sensory structure.

In the following, the fundamentals and the numerical modelling of the combined joining and forming process are described. Thereafter, the utilization of the sensor signals for process control is discussed and a testing of a sensory structure is presented.

\section{Process Principle}

The fusion step is performed by the cold forming process rotary swaging, Fig. 2. First, a preform is formed by using inner tools to reduce the wall-thickness of a tube (a). The recess tools form a small inner shoulder, which serves as a stop for the end cap. A deformation sensing element with chamfered end caps is inserted and integrated by a further forming process of the tubular part (b). An axial pretension is successively generated by the displaced material on the end cap (c). By this the desired sensory properties of the component are achieved. By contrast, it is not possible to generate a mere force fit of the end caps in radial direction, as the elastic springback after each forming stroke prevents a radial clamping.

The geometry of the preform and the end caps have to be designed to guarantee the desired material flow and to adjust the joining forces. For this, a numerical model is introduced. 


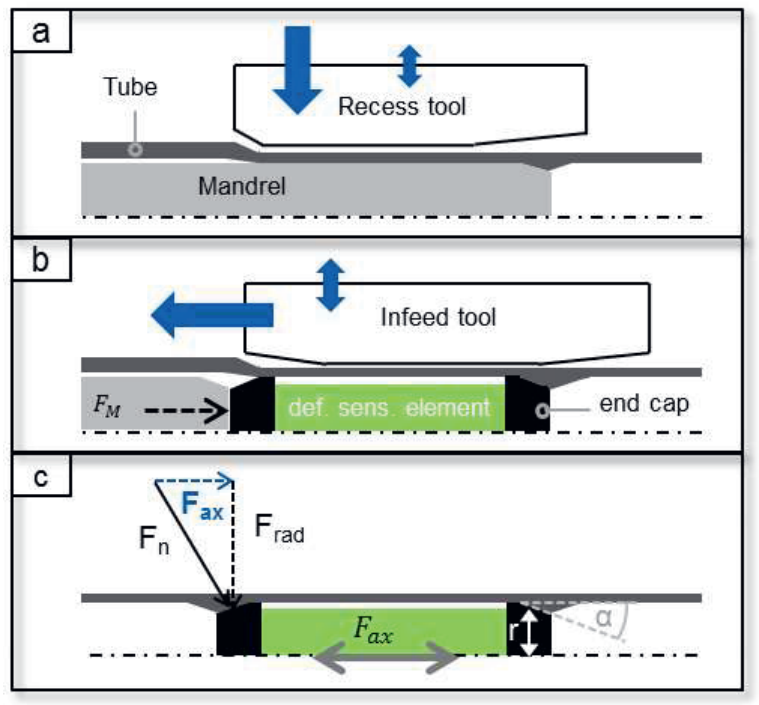

Fig. 2. Process steps to integrate and form sensory machine elements

\section{Numerical model}

A 3-dimensional model is required to simulate this specific forming process due to the asymmetric tool engagement [3]. The materials of the different bodies are modelled according to Fig. 3. The deformation sensing element is made of aluminum and equipped with $350 \mathrm{Ohm}$ strain gauges. A half bridge of two linear gauges (HBM LY4) on opposite sides is used to measure the axial pre-tension forces during forming. The tools are rotating around the workpiece and incrementally form the tubular pre-form with tool strokes of $29 \mathrm{~Hz}$. An implicit solver is used to calculate the elastic springback occurring after each contact. Instead of simulating the full length of the part $(250 \mathrm{~mm})$, only the essential joining zone is taken into account (24 $\mathrm{mm}$ from the first contact).

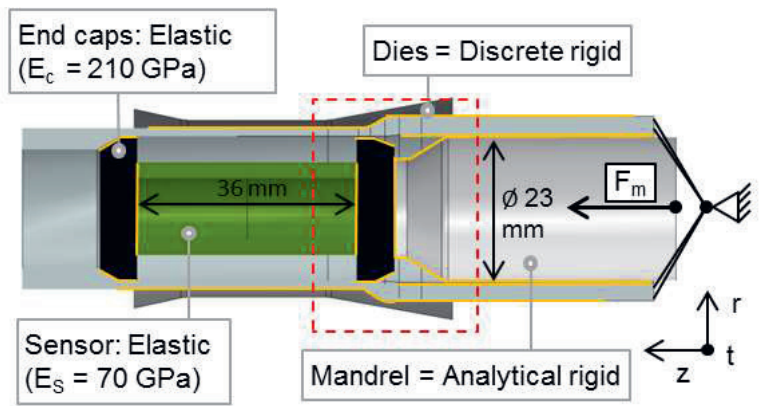

Fig. 3. Description of the numerical model

Further properties of the process, which have to be taken into account, are the cold-hardening of previous swaging operations, the temperature increase and the elasticity of the swaging machine. A more detailed description of the implementation of these features can be found in [2].
A calculated view-cut of the joining zone (marked red in Fig. 3) is illustrated in Fig. 4 (top). To compare the resulting geometry an $\mathrm{x}$ ray computer tomography scan of a successfully joined part is obtained, Fig. 4 (bottom).

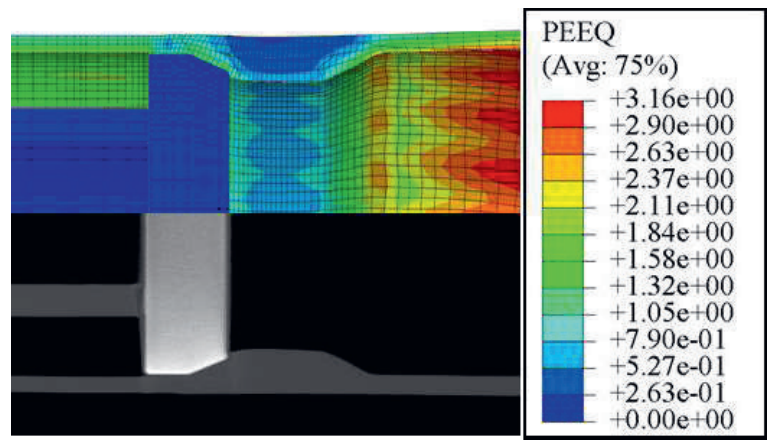

Fig. 4. Equivalent plastic strain distribution (top) and experimentally (bottom) determined geometry of the joining zone (marked red in Fig. 3)

The resulting joining forces of an experimental (in-situ measurement) and numerical integration process are compared, Fig. 5. Two materials, a standard extruded aluminum alloy EN AW 6060 and a mild steel SJ235 are investigated. Both, the geometry of the joining zone as well as the occurring forces are in good accordance. The remaining pre-tension force of the aluminum alloy is determined to $2,3 \mathrm{kN}$ numerically and $3.3 \mathrm{kN}$ experimentally. For steel the accordance is even higher $(6.7 \mathrm{kN}$ numerically to $7.1 \mathrm{kN}$ experimentally). The maximum process forces are around 2-3 times of the remaining pretension force. This has to be considered during the design of the deformation sensing element to avoid a plastification of the element during the joining process.

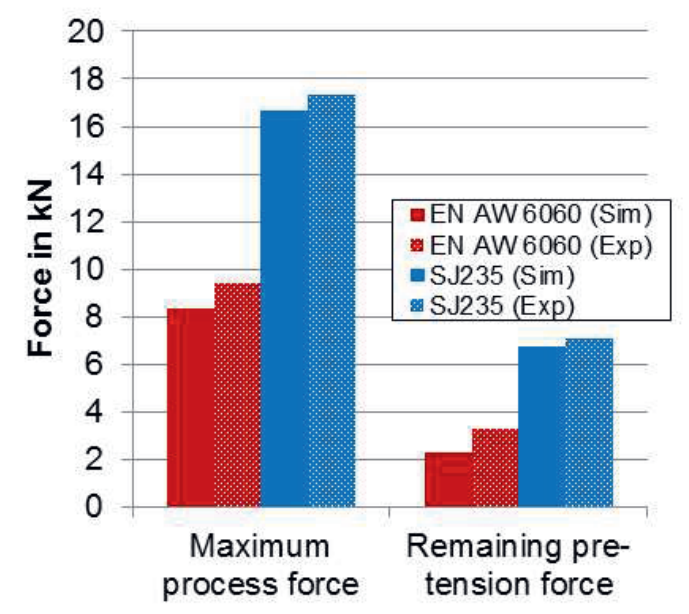

Fig. 5. Validation of forces of the numerical model 


\section{Process monitoring and control}

During the forming process, the sensor signals of the deformation element are acquired with $1 \mathrm{kHz}$. On the one hand, this is useful to validate the numerical simulations. On the other hand, these signals also contain valuable information for monitoring and control of the production processes. The signals are digitalized and wirelessly transmitted with an integrated sensor electronics board in the rotating tubular component, Fig. 6. To utilize the signals, they have to be calibrated roughly at the beginning of each process. For this, a preliminary calibration factor of the deformation sensing element is gained. A suitable reference force is the mandrel force $F_{M}$ (Fig. 2b) occurring at the first seconds of the process without superimpositions of forming forces.

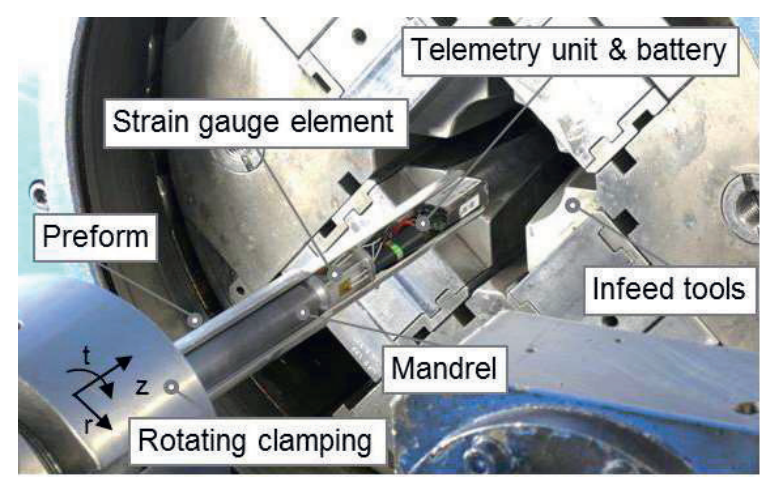

Fig. 6. Process monitoring and control by in-situ measurement of joining forces [2]

After the constant mandrel force is applied, the actual forming begins which induces force peaks on the deformation sensing element. The force course is recorded and analyzed for irregularities. Process faults such as cracks or wrong alignment are detected and the process can be stopped in order to sort out the faulty part.

However, even though the joining partners are aligned correctly and no faults are evident, small deviations in the resulting pre-tension force occur in any case. This is due to common fluctuations in the properties of the materials, the lubrication conditions and geometrical deviations within the tolerance limit. In order to achieve consistent sensor properties of the produced parts, an adaption of the process parameters is performed on the base of the acquired sensor signals, Fig. 7. The integration forces are measured. Each stroke of the tools generates a peak and shifts the corresponding plateau to another level (a). The envelopes of the peaks and valleys are identified. Discrete comparison points $1-4$ are determined and compared to reference values (b). A correcting factor is calculated to take countermeasures for parts leaving the targeted range. Ongoing investigations have proven an occurring time slot in which an interference of the joining process is possible. Process parameters such as infeed speed and mandrel force can be adapted online and influence the force course into the desired direction (c) [4].

a)

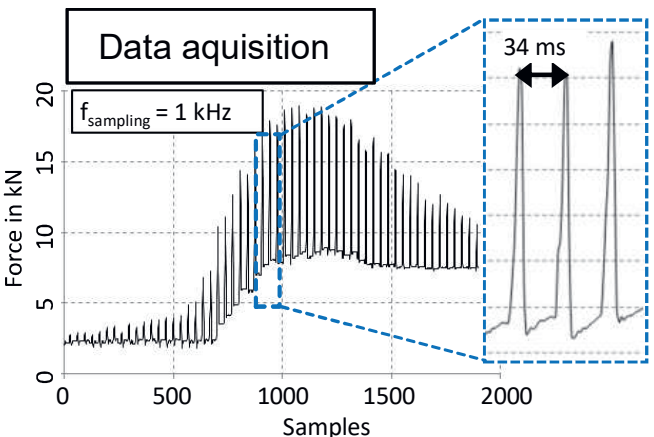

b)

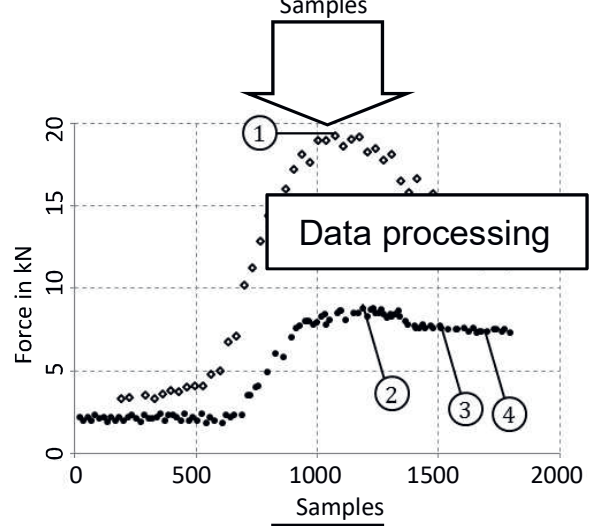

c)

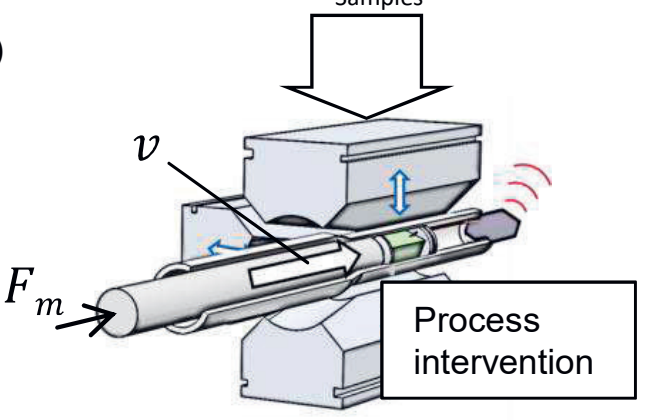

Fig. 7. Process control by in-situ measurement and processing of joining forces

A first control approach was implemented and evaluated. An improvement in accuracy of around $65 \%$ compared to the uncontrolled process could be achieved [4].

\section{Testing of a torque sensing shaft}

A $32 \times 4 \mathrm{~mm}$ torque measuring steel shaft is manufactured by this method. In contrast to the axial-force measurement mentioned before, a different bridge circuit is used to measure torque. Two v-shaped shear strain gauges with 2 measuring grids (HBM XY4) are attached to the deformation sensing element. The $350 \mathrm{Ohm}$ full bridge is fed with $2.5 \mathrm{~V}$ with the universal measurement amplifier Quantum MX840B by HBM. A torque testing machine (company Suter) is used, Fig. 8. A saw tooth pattern is 
applied in both load directions without zeroing in between. Due to the pre-tension the sensor shows a high linearity in the range of $\pm 1.2 \mathrm{kNm}$. The width of the hysteresis in its maximum accounts to $0.5 \%$, while the linearity error is $0.67 \%$.

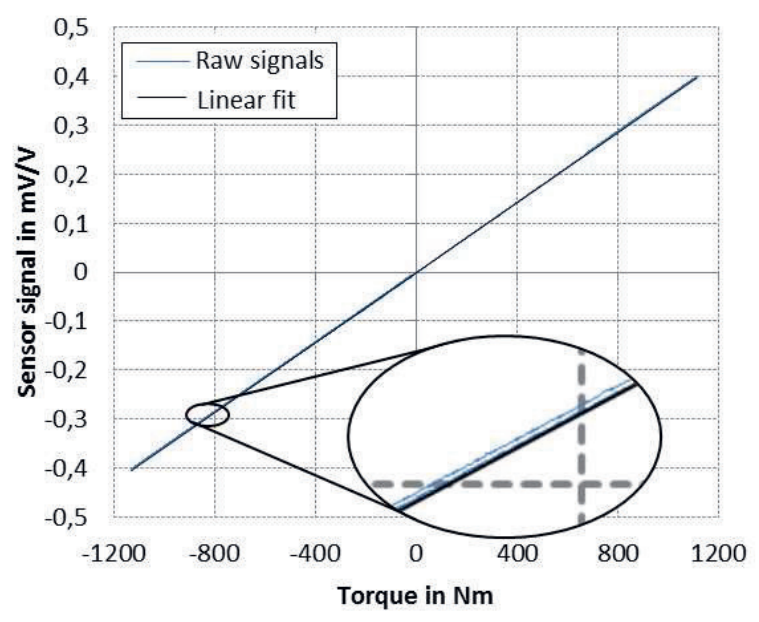

Fig. 8. Calibration curve of a sensory shaft, loaded with torque in saw tooth pattern

\section{Example application}

The presented integration method is applicable to a variety of industrial and domestic applications. Sensory and connected devices are on the advance due to availability of costeffective electronics. Fig. 9 demonstrates the integrated sensor approach in form of a sensory weight bar.

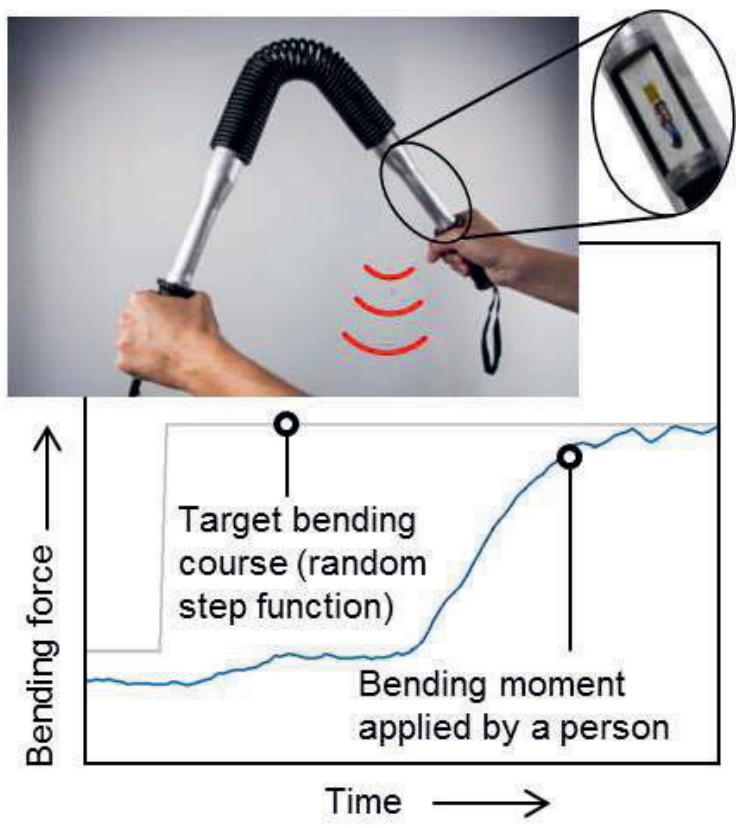

Fig. 9. Wireless sensory bending bar to improve strength and dexterity [4]

In this example, four linear strain gauges arranged at an angle of 90 degrees are attached to the deformation element. The strain gauges are wired in quarter-bridge individually and the resulting bending moment is calculated. The software provides an ideal set course which the user has to follow by bending the device.

\section{Conclusion}

A novel manufacturing route for the efficient production of mechanical sensors is presented. A deformation sensing element is joined with a force- and form-fit inside a tubular part during its forming process. This offers the advantage of utilizing the integrated sensors for process monitoring and control as well as an in-situ calibration. The design of the joining process is supported by numerical simulation. A good accordance of the calculated joining forces with measurements can be observed. A torque measuring deformation element inside a $32 \times 4$ steel tube was calibrated with $\pm 1.2 \mathrm{kNm}$. An exemplary application was demonstrated in form of a sensory bending bar and demonstrates the applicability of measuring in exposed mobile structures.

\section{Acknowledgements}

The authors thank the German Research Foundation (DFG) for the sponsorship of the sub-project B4 „Integration of functional materials" of the collaborative research center 805 "Control of Uncertainty in Load-Carrying Structures in Mechanical Engineering".

\section{References}

[1] Lohrengel, A., Schäfer, G., Rolshofen, W., Drehmomentmessung an einer Gelenkwelle im Antriebsstrang. IMW - Institutsmitteilung Nr. 34 (2009)

[2] P. Groche, M. Krech, Efficient production of sensory machine elements by a two-stage rotary swaging process - Relevant phenomena and numerical modelling. Journal of Materials Processing Technology 242, 205-217 (2017); doi: 10.1016/j.jmatprotec.2016.11.03

[3] L. Fan, Z. Wang, H. Wang, 3D finite element modeling and analysis of radial forging processes, Journal of Manufacturing Processes 16, 329-334 (2014); doi: 10.1016/j.jmapro.2014.01.005

[4] M. Krech, P. Groche, Controlling the sensor properties of smart structures produced by metal forming, International Conference on the Technology of Plasticity 2017 (submitted Paper) 